\title{
Estradiol and nicotine exposure enhances A549 bronchioloalveolar carcinoma xenograft growth in mice through the stimulation of angiogenesis
}

\author{
MICHAEL J. JARZYNKA ${ }^{1,3}$, PING GUO ${ }^{1,3,4}$, IFAT BAR-JOSEPH ${ }^{1,3}$, BO HU ${ }^{1,2}$ and SHI-YUAN CHENG ${ }^{1,3}$ \\ ${ }^{1}$ Cancer Institute, Departments of ${ }^{2}$ Medicine or ${ }^{3}$ Pathology, Research Pavilion at the Hillman Cancer Center, \\ University of Pittsburgh, 5117 Centre Avenue, Pittsburgh, PA 15213-1863, USA; \\ ${ }^{4}$ North China Coal Medical College, Tangshan, Hebei 063000, P.R. China
}

Received September 1, 2005; Accepted October 13, 2005

\begin{abstract}
Angiogenesis is required for lung cancer growth, which is mediated by various growth factors such as vascular endothelial growth factor (VEGF). Increases in VEGF and angiogenesis have been correlated with poor prognosis and survival in patients with lung cancer. In addition, recent reports show that estradiol and nicotine play important roles in lung tumor initiation and progression. In this report, we demonstrate that estradiol and nicotine exposure enhances the growth of A549 bronchioloalveolar carcinoma xenografts in mice through the stimulation of cell proliferation, VEGF secretion and angiogenesis. We detect a four-fold increase in microvascular density in tumors from mice exposed to estradiol and nicotine compared to control tumors resulting in an increase in tumor growth. Intriguingly, the effects on angiogenesis and tumor growth by the combination of agents were additive when compared to either agent alone. Furthermore, estradiol promotes VEGF secretion from various non-small cell lung carcinoma (NSCLC) cells and this effect is augmented by nicotine in a tumor xenograft model. These results indicate that aside from their roles in promoting cell proliferation, estradiol and nicotine appear to have additive effects on the induction of angiogenesis through the stimulation of VEGF secretion during NSCLC progression.
\end{abstract}

\section{Introduction}

Lung cancer is the leading cause of cancer deaths in the United States and continues to be a major problem throughout the world $(1,2)$. Currently, the number of yearly lung cancer deaths in women exceeds the combination of breast, ovarian

Correspondence to: Dr Shi-Yuan Cheng, Cancer Institute and Department of Pathology, Hillman Cancer Center, Research Pavilion, Suite 2.26f, University of Pittsburgh, 5117 Centre Avenue, Pittsburgh, PA 15213-1863, USA

E-mail: chengs@upmc.edu

Key words: estradiol, nicotine, lung tumor, angiogenesis, VEGF and uterine cancer deaths. Increasing evidence reveals that hormonal variations among the sexes can lead to differences in the development of lung cancers (3). Specifically, estrogens appear to play an important role in lung tumorigenesis. Recent reports have demonstrated the presence of functional estrogen receptors in lung cancer cell lines (4-7). Furthermore, estradiol has been shown to promote lung tumor cell proliferation in vitro and in vivo (4-7).

In solid tumors such as human lung cancers, angiogenesis is required for tumor growth and is responsible for assuring the delivery of oxygen and nutrients, removal of waste products and the facilitation of metastasis (8). Angiogenesis is a complex multi-step process involving the formation of new blood vessels from pre-existing vascular networks and plays important roles in lung cancer progression (9). Accumulated evidence demonstrates that a balance of pro- and anti-angiogenic factors regulates angiogenesis. An important activator in this process is vascular endothelial growth factor (VEGF)-A. VEGF is a specific, potent growth factor of endothelial cells increasing their proliferation and migration thereby promoting angiogenesis (10). In lung cancer, increases in VEGF levels, microvascular density and neovascularization have been correlated with poor patient prognosis and survival (11). Currently, a variety of anti-angiogenic agents are in clinical development to target VEGF and its signaling for the treatment of lung cancer (12). A variety of stimuli including hypoxia, growth factors, cytokines and steroid hormones such as estrogens regulate VEGF gene expression in normal and tumor cells (13-15). However, whether estradiol modulates angiogenesis through the stimulation of VEGF expression/ secretion in lung cancer cells has not been studied.

Smoking is the major cause in the development of lung cancer (16). A major component of tobacco smoke well known for its addictive properties is nicotine. In addition to its roles in tobacco dependence, nicotine increases lung tumor cell proliferation through the stimulation of nicotinic acetylcholine receptors and the Akt signaling pathway (17). Recent reports demonstrate that nicotine promotes tumor angiogenesis through the stimulation of several cell types that are important in tumor progression. For example, endothelial cells express functional nicotinic receptors (18) and stimulation of these receptors with nicotine induces 
DNA synthesis and cell proliferation (19). Furthermore, nicotine increases expression of VEGF in endothelial cells (20) and stimulates angiogenesis in a mouse tumor model (21) through the $\alpha 7$ nicotinic acetylcholine receptor (22). However, whether nicotine can further augment lung tumor angiogenesis in combination with estradiol is not known.

The goal of this study is to determine whether estradiol and nicotine act in conjunction in stimulating lung tumor angiogenesis. Here, we report that estradiol and nicotine exposure enhances the growth of A549 bronchioloalveolar carcinoma xenografts in mice through increases in cell proliferation, VEGF secretion and angiogenesis. To our knowledge, this is the first study to examine the effects of estradiol and nicotine in combination on lung tumor growth and angiogenesis. These findings may have important implications on current strategies for smoking cessation and treatment of lung cancers.

\section{Materials and methods}

Reagents and antibodies. B-estradiol and (-)-nicotine were obtained from Sigma-Aldrich (St. Louis, MO). 17- $\beta$-estradiol $\left(\mathrm{E}_{2}, 0.72 \mathrm{mg}, 60\right.$-day slow release pellets) were purchased from Innovative Research of America (Sarasota, FL). The following antibodies were used in our studies: goat anti-human Ki-67 polyclonal antibody (Santa Cruz Biotechnology, Santa Cruz, CA), rabbit anti-human VEGF-A polyclonal antibody (Santa Cruz), rat anti-mouse CD31 (PECAM-1) monoclonal antibody (BD BioSciences Pharmingen, San Jose, CA), mouse anti-human VEGF monoclonal antibody (BD BioSciences Pharmingen) and goat anti-human $\beta$-actin polyclonal antibody (Santa Cruz). Other materials and reagents were from Fisher Scientific (Pittsburgh, PA), Invitrogen (Grand Island, NY) or Sigma.

Cell culture. The following cell lines were utilized in our studies. Human A549 bronchioloalveolar carcinoma and H23 lung adenocarcinoma cells were obtained from American Type Culture Collection (Rockville, MD). The 128-88T, 91T and 784T primary non-small cell lung carcinoma (NSCLC) cell lines were a kind gift from Dr Jill M. Siegfried (University of Pittsburgh Cancer Institute, Pittsburgh, PA) (4-7). The cells were cultured in DMEM (Invitrogen) supplemented with $10 \%$ FBS (Hyclone, Salt Lake City, UT) and $1 \%$ penicillin-streptomycin (Sigma) or in phenol-red free DMEM supplemented with 1-10\% charcoal-stripped serum (CSS, Hyclone).

A549 bronchioloalveolar carcinoma xenografts. A549 cells $\left(1 \times 10^{7}\right.$ cells) were suspended in PBS and subcutaneously implanted into the flank of 6 week-old ovariectomized female nude mice (Harlan, Indianapolis, IN) supplemented with or without $\mathrm{E}_{2}$ pellets. In addition, certain mice received nicotine (200 $\mu \mathrm{g} / \mathrm{ml}$ ) via their drinking water. The mice were divided into four experimental groups consisting of 8 mice per group: i) control, ii) nicotine, iii) $E_{2}$ and iv) $E_{2}$ plus nicotine. Tumor volume was estimated every five days using width $(a)$ and length $(b)$ measurements $\left(a^{2} b / 2\right.$, where $\left.a<b\right)$. At the end of the experiments, the tumors were excised, embedded in OCT, sectioned and stored at $-80^{\circ} \mathrm{C}(23)$.
Immunohistochemical staining. Immunohistochemical (IHC) analyses were performed to assess microvascular density (MVD), cell proliferation and VEGF secretion using antiCD31 (1:1000), anti-Ki-67 (1:500) and anti-VEGF (1:500) antibodies. All of the primary antibody reaction products were subsequently visualized with their respective biotinylated secondary antibodies. The antibody complexes were developed with DAB and counterstained. The images of antibody staining from serial sections in each treatment group were captured using an Olympus BX51 microscope (Melville, NY) equipped with a digital camera (24).

Quantitative analyses of IHC staining. Quantitative analyses for MVD and proliferating nuclei were performed as previously described (23). The MVD was calculated as the ratio of positively stained areas to the total area of the field. The proliferation index of the A549 xenografts was assessed in a similar manner and calculated as a percentage of Ki-67positive nuclei (brown color) to the total number of nuclei (blue color) within the same microscopic field.

In vitro cell proliferation assay. The effect of estradiol and/or nicotine on A549 cell proliferation was determined by the Biotrak cell proliferation ELISA system (Amersham Pharmacia Biotech, Piscataway, NJ). Briefly, A549 cells $\left(3 \times 10^{3}\right)$ were seeded into 96-well plates with DMEM containing $10 \%$ FBS. Twenty-four hours later, the cells were washed, and the medium was changed to serum-free/phenol red-free DMEM with or without increasing concentrations of estradiol (0.1, $10,50$ and $100 \mathrm{nM})$ and/or nicotine (100 nM, $500 \mathrm{nM}, 1 \mu \mathrm{M}$ or $10 \mu \mathrm{M}$ ) for $72 \mathrm{~h}$. BrdUrd was added into the wells during the last two hours of the incubation period. Afterwards, the proliferation of the cells was measured according to the manufacturer's instructions. Data were normalized against the vehicle-treated control cells and expressed as percent increase in BrdUrd incorporation.

In vitro VEGF secretion. The effect of estradiol and/or nicotine on the secretion of VEGF from NSCLC cells was assessed using a Quantikine human VEGF immunoassay kit (R\&D Systems, Inc., Minneapolis, MN) as previously described (23). Briefly, human NSCLC cells $\left(2.5 \times 10^{5}\right)$ were seeded into 12 -well plates with DMEM containing 10\% FBS. The next day, the cells were washed, and the medium was changed to serum-free/phenol red-free DMEM for $24 \mathrm{~h}$. After this period, the cells were exposed to phenol red-free DMEM containing $1 \% \mathrm{CSS}$ with or without increasing concentrations of estradiol $(0.1,1$ and $10 \mathrm{nM})$ and/or nicotine $(100 \mathrm{nM}, 500 \mathrm{nM}, 1 \mu \mathrm{M}$ or $10 \mu \mathrm{M})$. After $48 \mathrm{~h}$, VEGF in the collected conditioned media (CM) was measured according to the manufacturer's instructions. To rule out the effect of increased tumor cell proliferation, the VEGF concentration was first normalized to the total cell number for each estradiol concentration. Afterwards, the data were normalized against the vehicletreated control cells and expressed as percent increase in VEGF secretion.

Western blot analysis. Tumor tissue from each treatment group was suspended in RIPA buffer containing protease inhibitors and lysed. Afterwards, the tissue lysates were centrifuged, 
A

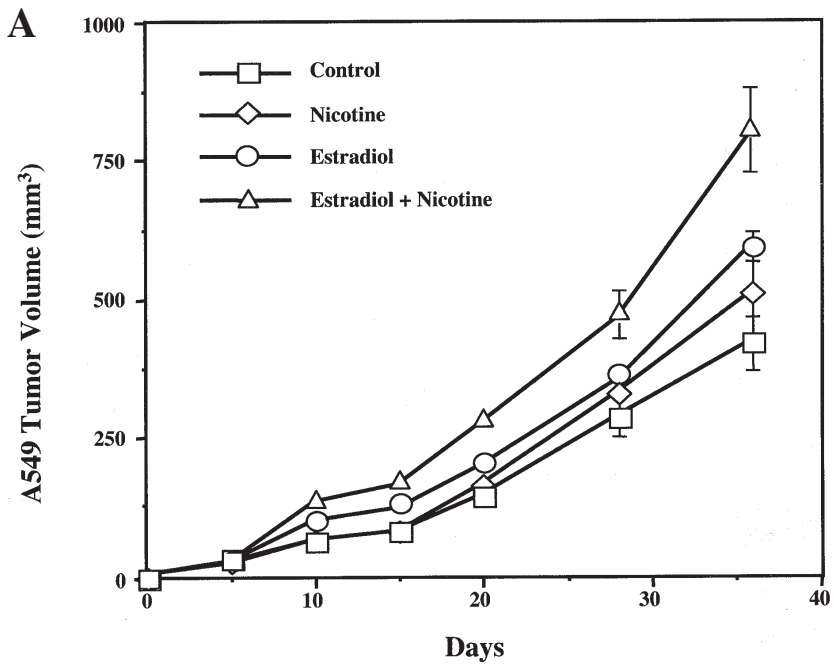

B

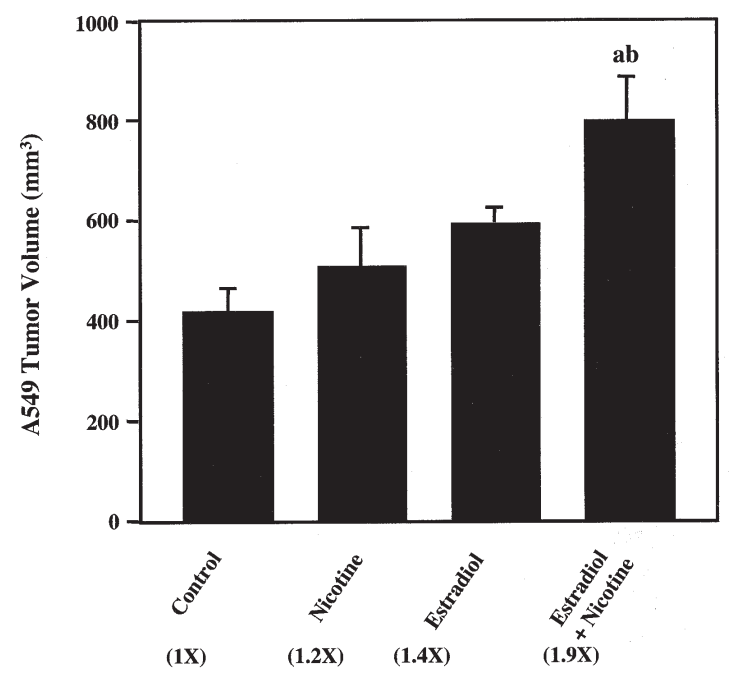

Figure 1. Estradiol and nicotine stimulate the growth of A549 bronchioloalveolar carcinoma xenografts in mice. (A), A549 tumor growth kinetics. A549 cells $\left(1 \times 10^{7}\right.$ cells) were subcutaneously implanted into the flank of mice supplemented with or without estradiol (0.72 mg, 60-day slow release pellets) and/or nicotine $(200 \mu \mathrm{g} / \mathrm{ml}$ via drinking water). Tumor volumes were measured every five days. (B), End-point A549 tumor volume. Numbers in parentheses represent the fold increase compared to control tumors. Data were analyzed by One-way ANOVA followed by a Newman-Keuls post-hoc. Letters above the bars indicate: (a), p $<0.05$ when compared to control; or (b), nicotine-treated animals. Data in (A) and (B) represent the mean \pm SEM of 2 independent experiments with 8 to 10 mice per group.

supernatants were collected and equal amounts of total protein $(30 \mu \mathrm{g})$ were separated on a SDS/10\% polyacrylamide gel then transferred to an Immobilon-P membrane (Millipore, Bedford, MA). The membrane was subjected to Western blot analysis using a mouse monoclonal anti-VEGF antibody $(1: 1000)$ and a goat anti-ß-actin (1:2000) antibody as previously described (25).

Statistical analysis. Statistical analyses were performed using Student's t-test or a One-way ANOVA followed by a Newman-Keuls post-hoc. A $\mathrm{p}<0.05$ was considered statistically significant. Data were expressed as the mean \pm the standard deviation (SD) or the standard error of the mean (SEM).

\section{Results}

Estradiol and nicotine enhance A549 bronchioloalveolar carcinoma growth in mice. A549 cells were subcutaneously implanted into the flanks of ovariectomized nude mice with or without exposure to estradiol, nicotine or estradiol plus nicotine. At 36 days post-implantation, mice that received the combination of estradiol and nicotine developed significantly larger A549 tumors (Fig. 1A), with a final tumor volume of $800 \pm 86.1 \mathrm{~mm}^{3}$ compared to $418 \pm 50.4 \mathrm{~mm}^{3}$ for the control animals (Fig. 1B). In contrast, there was no significant difference in tumor volume between the estradiol- $(595 \pm 29.6$ $\left.\mathrm{mm}^{3}\right)$ and nicotine-treated $\left(507 \pm 79.6 \mathrm{~mm}^{3}\right)$ groups compared to the controls $\left(418 \pm 50.4 \mathrm{~mm}^{3}\right)$. Interestingly, the estradiol and nicotine combination enhanced tumor growth within 10 days of tumor cell implantation (Fig. 1A). Equally, differences in tumor weights paralleled the fold changes as seen in the tumor volume measurements (data not shown). Thus, the combination of estradiol and nicotine exposure promoted A549 bronchioloalveolar carcinoma growth in mice.

Estradiol and nicotine enhance tumor cell proliferation in vitro and in vivo. Next, we determined the effects of estradiol and/or nicotine on the cell proliferation of A549 tumors from the various treated mice using Ki-67 IHC. Mice that were exposed to nicotine alone (Fig. 2A, panel b) or estradiol alone (Fig. 2A, panel c) demonstrated a higher percentage of Ki-67-positive nuclei versus the controls (Fig. 2A, panel a). Quantification of the IHC for Ki-67 revealed a >2-fold increase in proliferating nuclei (Fig. 2B). More importantly, the combination of estradiol and nicotine lead to an augmentation of cell proliferation compared to either agent alone (Fig. 2A, panel d). This result demonstrated a $>4$-fold increase in proliferation when compared to control mice (Fig. 2B).

We examined whether estradiol, nicotine or their combination would promote A549 cell proliferation in vitro. Fig. $2 \mathrm{C}$ shows that estradiol alone stimulated A549 cell proliferation at concentrations ranging from 1 to $100 \mathrm{nM}$. Interestingly, nicotine exposure did not promote BrdUrd incorporation into these cells. In concentrations above $10 \mu \mathrm{M}$, nicotine had detrimental effects leading to the detachment of A549 cells from the cell culture plates (data not shown). Moreover, the addition of nicotine with estradiol did not further promote A549 cell proliferation when compared to estradiol alone (Fig. 2C).

Estradiol and nicotine enhance microvascular density (MVD) within A549 tumor xenografts. Next, we performed IHC analyses on various tumor sections to assess whether mice that were exposed to estradiol, nicotine or both agents demonstrated an increase in angiogenesis in the A549 tumor xenografts. As shown in Fig. 3A, nicotine (panel b), estradiol (panel c) and the combination of estradiol and nicotine (panel d) enhanced the MVD in A549 tumors compared to controls (panel a) when qualitatively examining the CD31 IHC staining. However, quantitative analysis showed that although the nicotine group demonstrated an upward trend in MVD, only the estradiol group and the combination group had statistically significant increases in MVD when compared to controls (Fig. 3B). Mice that were exposed to the combination 
A
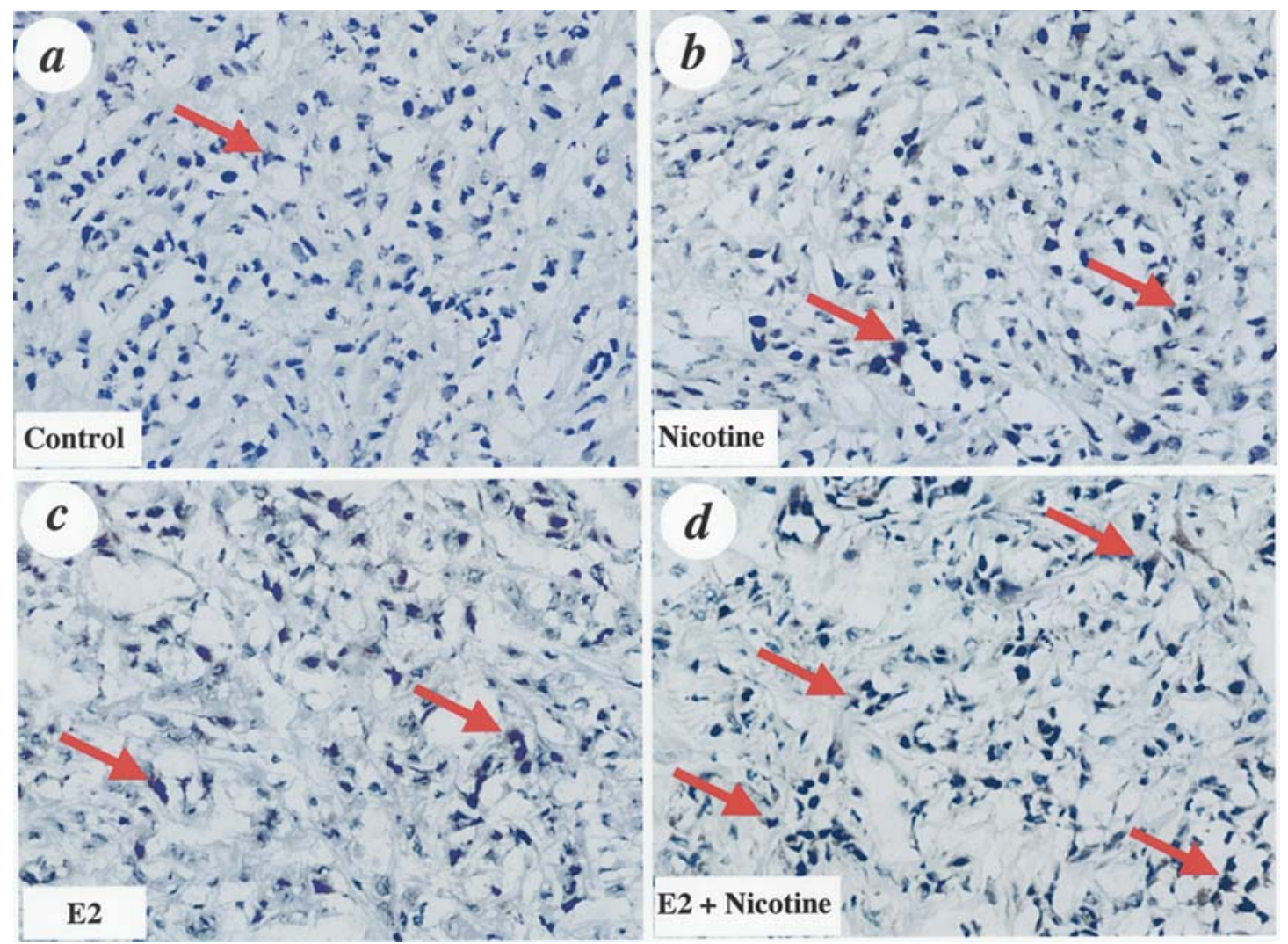

B

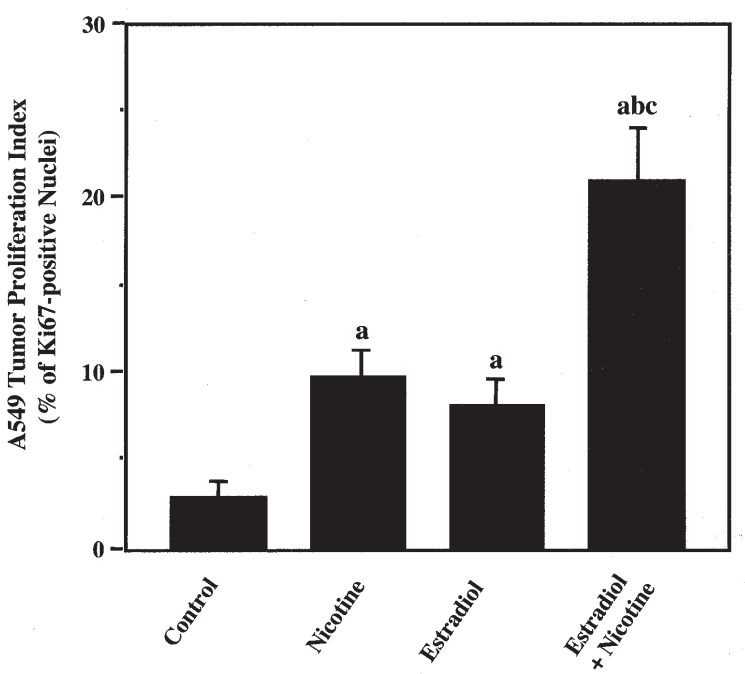

C

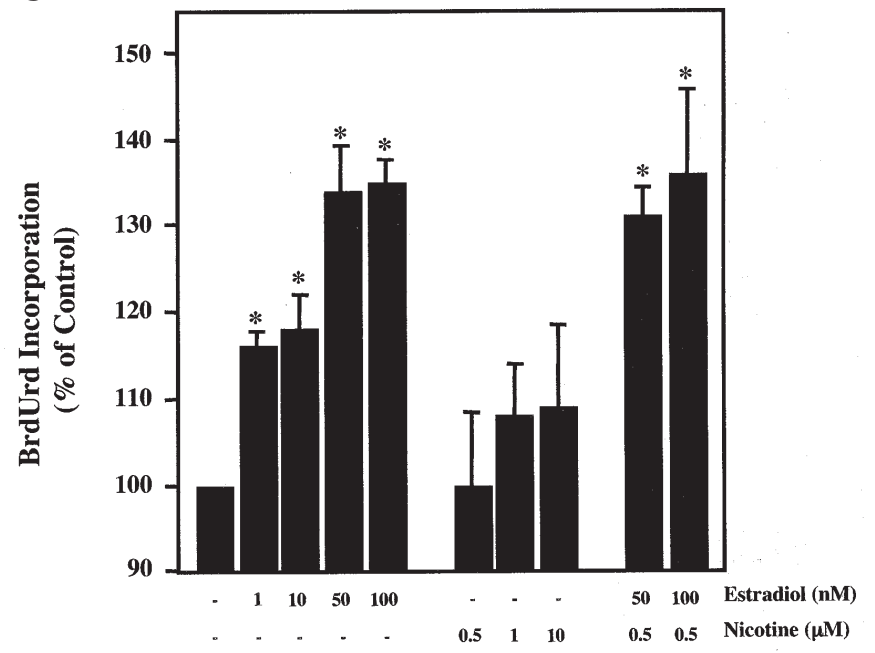

Figure 2. Estradiol and nicotine stimulate A549 cell proliferation. (A), IHC analyses of control (a), nicotine-treated (b), estradiol-treated (c) and estradiol and nicotine-treated (d) A549 tumors. Arrows indicate Ki-67-positive nuclei. Three to five serial sections from 8 individual tumor samples from each group were independently analyzed. The experiments were repeated twice with similar results. Original magnification x400. (B), Quantification of Ki-67-positive nuclei. Data represent the mean \pm SEM of 2 independent experiments with 8 mice per group. Data were analyzed by One-way ANOVA followed by a NewmanKeuls post-hoc. Letters above the bars indicate: (a), $\mathrm{p}<0.05$ when compared to control; (b), nicotine-treated; or (c), estradiol-treated animals. (C), BrdUrd incorporation into A549 cells treated with increasing concentrations of estradiol and/or nicotine. Data represent the mean \pm SD of 3 or more independent experiments and were normalized against the vehicle-treated control cells. Symbol above the bars indicates: ${ }^{*}$ p $<0.05$ when compared to vehicle-treated cells determined by Student's t-test.

of estradiol and nicotine had a 4-fold higher MVD versus controls. The combination of agents resulted in a 2.2- and 1.6-fold enhancement in MVD in the A549 tumors when compared to nicotine alone and estradiol alone, respectively (Fig. 3B). These results suggest that estradiol and nicotine act in concert to stimulate angiogenesis in vivo.
Estradiol increases VEGF secretion from A549 tumor cells. To determine a potential mechanism for the increased tumor growth and angiogenesis due to estradiol and nicotine exposure in the tumor xenografts, we performed IHC analyses to examine VEGF expression in the various A549 tumors. As shown in Fig. 4A, estradiol (panel c), nicotine (panel b) and the 
A

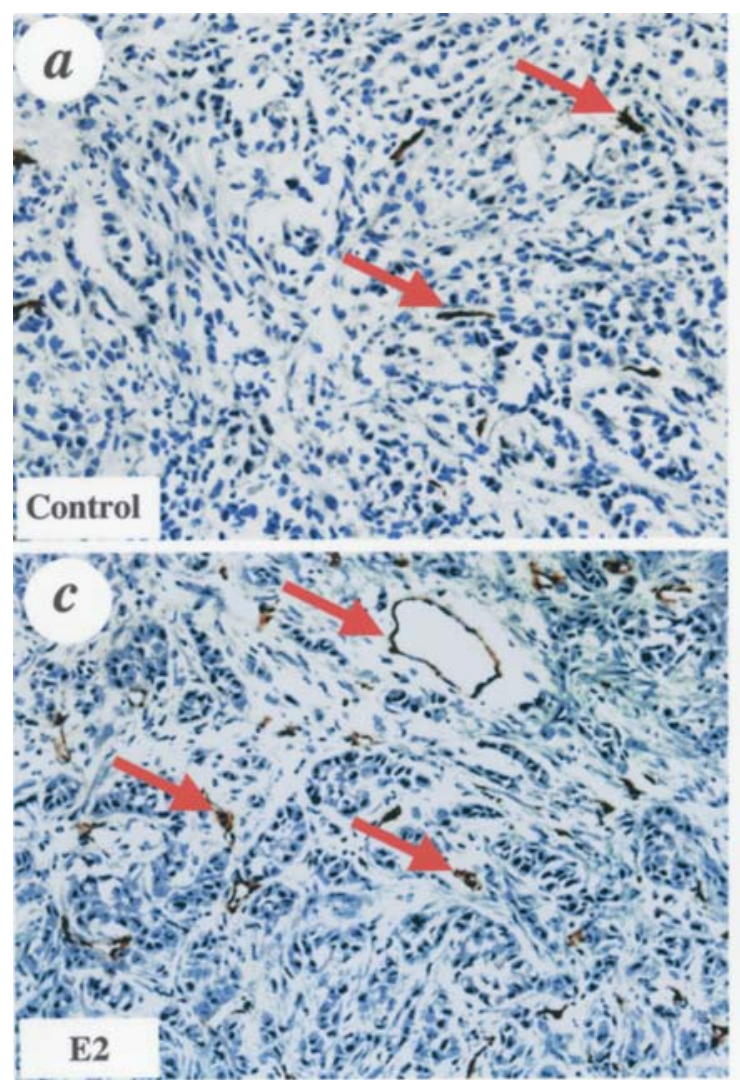

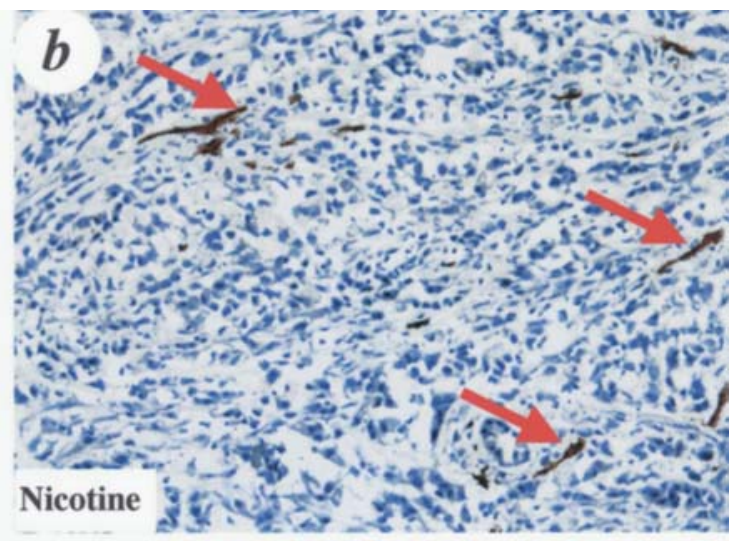

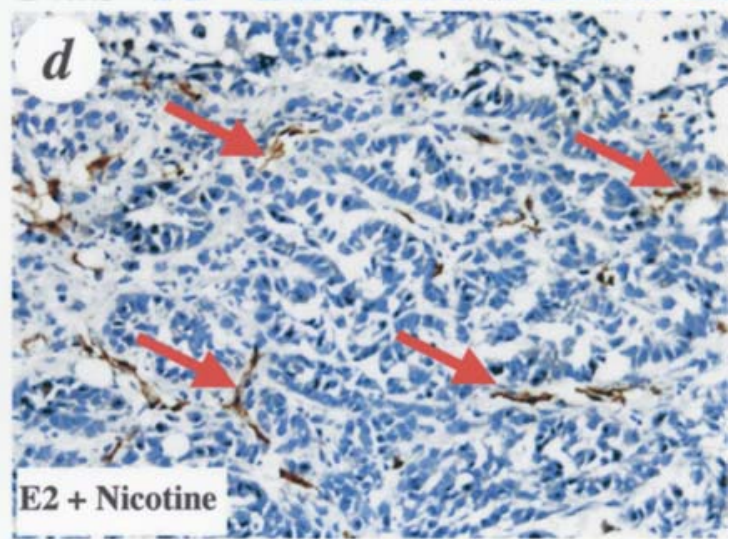

B

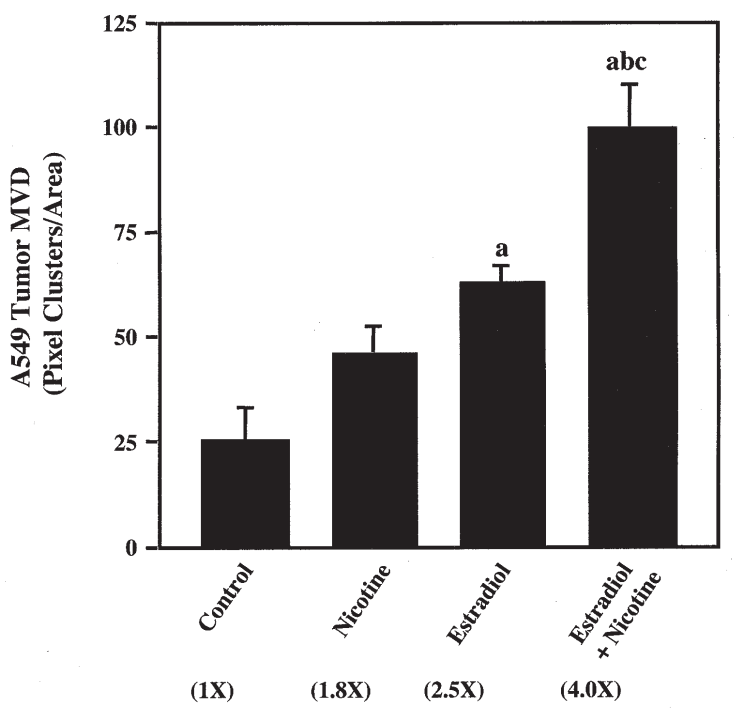

combination of estradiol and nicotine (panel d) enhanced VEGF expression in the A549 tumors compared to controls (panel a). Qualitative assessment of the IHC staining showed that the combination of estradiol and nicotine resulted in the greatest intensity of VEGF staining (Fig. 4A, panel d).

To confirm these IHC results, we performed Western blotting for VEGF on tissue lysates prepared from the various A549 tumors. As shown in Fig. 4B, nicotine, estradiol and the combination of estradiol and nicotine revealed an increase in VEGF expression compared to controls. This trend corroborates with the IHC data showing only a slight increase in VEGF expression in the nicotine-treated versus control xenografts. Estradiol elicited a greater stimulation of VEGF while the combination of estradiol and nicotine resulted in the highest level of VEGF expression.
Figure 3. Estradiol and nicotine stimulate A549 tumor angiogenesis. (A), IHC analyses of control (a), nicotine-treated (b), estradiol-treated (c) and estradiol- and nicotine-treated (d) A549 tumors. Arrows indicate CD31positive vessels. The representative IHC stains were from three to five serial sections from 8 individual tumor samples from each group. The experiments were repeated twice with similar results. Original magnification x200. (B), Quantification of CD31-positive vessels. Numbers in parentheses represent the fold increase compared to control tumors. Data were analyzed by oneway ANOVA followed by a Newman-Keuls post-hoc. Letters above the bars indicate: (a), $\mathrm{p}<0.05$ when compared to control; (b), nicotine-treated or (c) estradiol-treated animals. Numbers in the parentheses indicate: foldincrease compared to controls. Data represent the mean \pm SEM of 2 independent experiments with 8 mice per group.

To explore if this phenomenon was a universal effect seen in various NSCLC cell lines, we performed a VEGF ELISA on CM collected from A549, H23, 128-88T, 91T and $784 \mathrm{~T}$ NSCLC cell lines. We found an increase in VEGF concentration in the $\mathrm{CM}$ of all of the aforementioned cell lines in response to exposure to estradiol (Fig. 4C). The stimulatory effects of estradiol on VEGF secretion saturated at the $10 \mathrm{nM}$ concentration in all of the NSCLC cells. Only the $\mathrm{H} 23$ cell line was statistically responsive to the lowest concentration $(0.1 \mathrm{nM})$ of estradiol. Surprisingly, nicotine alone or in combination with estradiol had no stimulatory effect on VEGF secretion in the NSCLC cell lines when compared to vehicle-treated cells (data not shown). These data suggest that estradiol stimulates the secretion of VEGF from human NSCLC cell lines. 
A
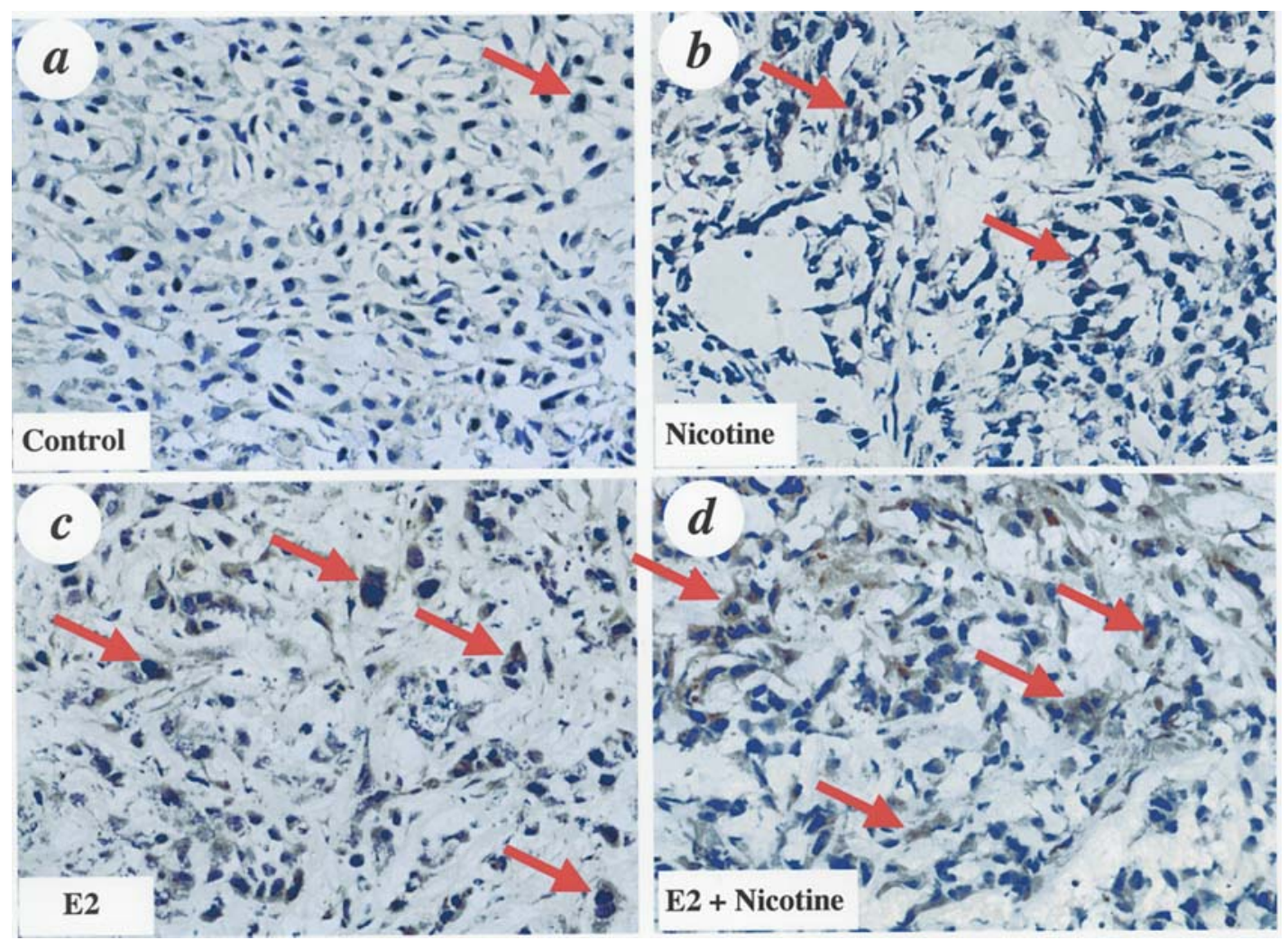

B

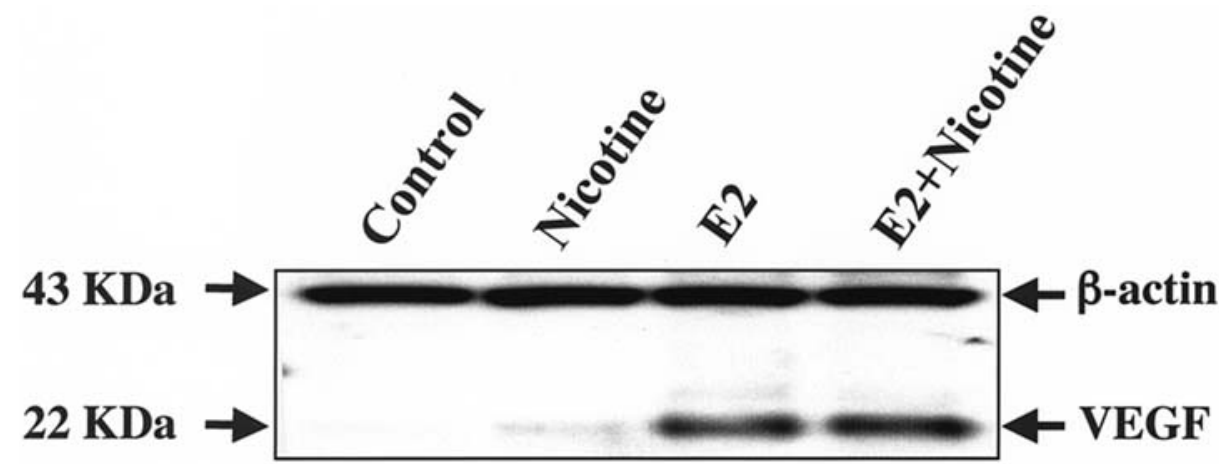

C

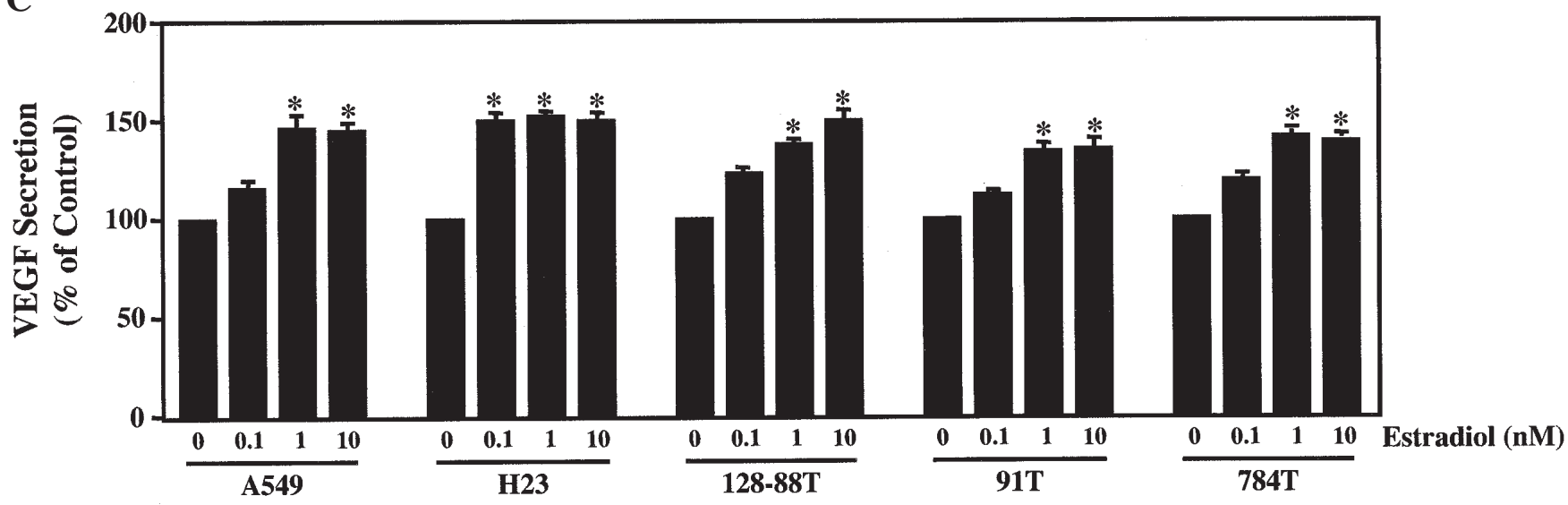

Figure 4. Estradiol and nicotine promote VEGF secretion from NSCLC tumor cells in vivo and in vitro. (A), IHC analyses of control (a), nicotine-treated (b), estradiol-treated (c) and estradiol and nicotine-treated (d) A549 tumors. Three to five serial sections from 8 individual tumor samples from each group were independently analyzed. The experiments were repeated twice with similar results. Original magnification x400. (B), Western blot analysis of lysates from control, nicotine-treated, estradiol-treated and estradiol- and nicotine-treated A549 tumor xenografts. The experiment was repeated two additional times with similar results. (C), VEGF ELISA analyses on CM collected after a 48-h culture of A549, H23, 128-88T, 91T and 784T tumor cells treated with increasing concentrations of estradiol. VEGF concentration was first normalized to the total cell number for each estradiol concentration. Afterwards, the data were normalized against the vehicle-treated control cells and expressed as percent increase in VEGF secretion. Data represent the mean \pm SEM of 3 or more independent experiments. Symbol above the bars indicates: * $p<0.05$ when compared to vehicle-treated cells determined by Student's t-test. 


\section{Discussion}

Estradiol has been shown to directly promote the proliferation of endothelial cells $(26,27)$ and VEGF expression in breast cancer cells (28-31), thereby stimulating angiogenesis. However, the effects of estradiol on lung tumor angiogenesis have not been determined. In this study, we report that estradiol stimulated angiogenesis in A549 bronchioloalveolar carcinoma xenografts demonstrating a 2.5 -fold increase in tumor MVD when compared to control mice. Additionally, estradiol promoted VEGF expression in various NSCLC cell lines in vitro. Furthermore, mice that were supplemented with estradiol developed A549 tumors with increased VEGF expression compared to mice without supplementation. To the best of our knowledge, this is a novel finding showing that estradiol stimulates the secretion of a major angiogenic factor in NSCLC cells.

In addition to enhancing angiogenesis and VEGF secretion, estradiol directly induces the proliferation of lung tumor cell lines in vitro and in vivo (5), through the stimulation of estrogen receptors that are present in NSCLC cell lines $(6,7)$. Importantly, ERß receptors that are expressed in both normal and cancerous lung tissue possess functionality when stimulated with estradiol (4). We observed that estradiol promoted A549 cell proliferation in vitro and augmented the number of proliferating nuclei in A549 tumor xenografts corroborating these recent studies. Taken together, the above results indicate that estradiol has multiple functions such as promotion of tumor cell proliferation and stimulation of angiogenesis, illustrating its important roles in lung tumor progression.

Previous studies indicate that nicotine stimulates vascular endothelial cell DNA synthesis, proliferation (19) and endothelial cell tube-like formation (21) in vitro. In addition, nicotine $(100 \mu \mathrm{g} / \mathrm{ml})$ delivered to mice via their drinking water has been shown to promote angiogenesis in multiple models such as hind limb ischemia reperfusion, artherosclerotic plaque growth and Lewis lung carcinoma growth (21). In our study, nicotine $(100 \mu \mathrm{g} / \mathrm{ml})$ was unable to promote A549 tumor growth in mice (data not shown). However, an increase in the nicotine dose to $200 \mu \mathrm{g} / \mathrm{ml}$ slightly stimulated A549 lung tumor growth and angiogenesis in comparison to control tumors albeit to a lesser degree than estradiol. Nicotine exposure resulted in a 1.8-fold increase in tumor MVD while modestly increasing VEGF expression when compared to control mouse tumors.

In our system, we observed that increasing concentrations of nicotine were unable to increase BrdUrd incorporation in vitro in the A549 cells, even though these cells express the $\alpha 7$ nicotinic acetylcholine receptor (32). Similar results were reported showing that nicotine did not stimulate Lewis lung carcinoma cell proliferation in vitro (21). We observed an increase in the proliferation index (Ki-67 staining) of A549 tumors from nicotine-treated mice when compared to control tumors. It is plausible that active metabolites of nicotine and/or factors in the milieu of the tumor microenvironment in vivo that are not present in the cell culture may have contributed to these results. Cotinine, a major metabolite of nicotine was demonstrated to be more potent than nicotine in stimulating the up-regulation of VEGF in endothelial cells
(20). Since VEGF is a potent mitogen, it is reasonable to believe that the enhanced cellular proliferation in nicotinetreated tumor xenografts was a result of VEGF-stimulated tumor angiogenesis. Intriguingly, VEGF has recently been shown to promote lung cancer cell proliferation (33). Thus, the nicotine-induced VEGF is probably responsible for the enhanced tumor and/or endothelial cell proliferation in these tumor xenografts.

Although recent reports demonstrate that estradiol and nicotine play important roles in lung tumor progression and angiogenesis, whether these two agents act in an additive or synergistic fashion in inducing angiogenesis has yet to be determined. In this study, we show that the combination of estradiol and nicotine enhanced NSCLC tumor volume to a greater degree than either compound alone. We present that estradiol and nicotine exposure resulted in a 4-fold increase in tumor MVD compared to control tumors, thus demonstrating an additive effect on tumor angiogenesis. Moreover, the combination of agents resulted in the most robust increase in VEGF secretion in vivo when compared to control, nicotine- and estradiol-treated A549 tumors. Interestingly, estradiol and nicotine did not result in an additive effect on VEGF secretion in vitro when compared to estradiol alone in the NSCLC cell lines tested. These additive effects of estradiol and nicotine on angiogenesis are likely the result of multiple mechanisms such as the promotion of endothelial cell proliferation and up-regulation of pro-angiogenic growth factors such as VEGF in the in vivo tumor microenvironment.

In conclusion, our results show that the combination of estradiol and nicotine has an additive effect on lung tumor angiogenesis through the stimulation of VEGF secretion thus enhancing the growth of A549 tumor xenografts. It appears that estradiol and nicotine functionally cooperate on the tumor and stromal compartments by having direct effects on tumor and endothelial cell proliferation and expression of angiogenic factors. Considering that increases in VEGF levels and angiogenesis have been correlated with poor patient prognosis and survival in lung cancer, this study provides further evidence illustrating the important roles that estrogen and nicotine take part in during lung tumor progression. Future lung cancer therapies for women may include the use of anti-estrogens similar to current treatments for breast cancer. Furthermore, all patients afflicted with lung cancer should be strongly encouraged to stop smoking not only to avoid the continual exposure to tobacco smoke carcinogens, but also possibly due to the pro-angiogenic effects of nicotine.

\section{Acknowledgements}

This study was supported in part by a Developmental Research Award (S.Y. Cheng) and a Career Development Award (M.J. Jarzynka) from the National Cancer Institute Specialized Programs of Excellence in Lung Cancer (P50 CA90440) awarded to the University of Pittsburgh Cancer Institute. We would like to thank Dr Pamela Hershberger for her helpful discussions and critical reading of this manuscript.

\section{References}

1. Patel JD, Bach PB and Kris MG: Lung cancer in US women: a contemporary epidemic. JAMA 291: 1763-1768, 2004. 
2. Devesa SS, Bray F, Vizcaino AP and Parkin DM: International lung cancer trends by histologic type: male:female differences diminishing and adenocarcinoma rates rising. Int J Cancer 117: 294-295, 2005.

3. Patel JD: Lung cancer in women. J Clin Oncol 23: 3212-3218, 2005.

4. Omoto Y, Kobayashi Y, Nishida K, Tsuchiya E, Eguchi H, Nakagawa K, Ishikawa Y, Yamori T, Iwase H, Fujii Y, Warner M, Gustafsson JA and Hayashi SI: Expression, function, and clinical implications of the estrogen receptor beta in human lung cancers. Biochem Biophys Res Commun 285: 340-347, 2001.

5. Stabile LP, Davis AL, Gubish CT, Hopkins TM, Luketich JD, Christie N, Finkelstein S and Siegfried JM: Human non-small cell lung tumors and cells derived from normal lung express both estrogen receptor alpha and beta and show biological responses to estrogen. Cancer Res 62: 2141-2150, 2002.

6. Hershberger PA, Vasquez AC, Kanterewicz B, Land S, Siegfried JM and Nichols M: Regulation of endogenous gene expression in human non-small cell lung cancer cells by estrogen receptor ligands. Cancer Res 65: 1598-1605, 2005.

7. Stabile LP, Lyker JS, Gubish CT, Zhang W, Grandis JR and Siegfried JM: Combined targeting of the estrogen receptor and the epidermal growth factor receptor in non-small cell lung cancer shows enhanced antiproliferative effects. Cancer Res 65: 1459-1470, 2005.

8. Folkman J: The role of angiogenesis in tumor growth. Semin Cancer Biol 3: 65-71, 1992.

9. Carmeliet P and Jain RK: Angiogenesis in cancer and other diseases. Nature 407: 249-257, 2000.

10. Ferrara N, Gerber HP and Le Couter J: The biology of VEGF and its receptors. Nat Med 9: 669-676, 2003.

11. Herbst RS, Onn A and Sandler A: Angiogenesis and lung cancer: prognostic and therapeutic implications. J Clin Oncol 23: 3243-3256, 2005.

12. Kerbel RS: Antiangiogenic drugs and current strategies for the treatment of lung cancer. Semin Oncol 31: 54-60, 2004

13. Loureiro RM and D'Amore PA: Transcriptional regulation of vascular endothelial growth factor in cancer. Cytokine Growth Factor Rev 16: 77-89, 2005

14. Hyder SM and Stancel GM: Regulation of VEGF in the reproductive tract by sex-steroid hormones. Histol Histopathol 15: 325-334, 2000.

15. Perrot-Applanat M, Ancelin M, Buteau-Lozano H, Meduri G and Bausero P: Ovarian steroids in endometrial angiogenesis. Steroids 65: 599-603, 2000.

16. Alberg AJ, Brock MV and Samet JM: Epidemiology of lung cancer: looking to the future. J Clin Oncol 23: 3175-3185, 2005.

17. Tsurutani J, Castillo SS, Brognard J, Granville CA, Zhang C, Gills JJ, Sayyah J and Dennis PA: Tobacco components stimulate Akt-dependent proliferation and NF\{kappa\}B-dependent survival in lung cancer cells. Carcinogenesis 26: 1182-1195, 2005.

18. Macklin KD, Maus AD, Pereira EF, Albuquerque EX and Conti-Fine BM: Human vascular endothelial cells express functional nicotinic acetylcholine receptors. J Pharmacol Exp Ther 287: 435-439, 1998.

19. Villablanca AC: Nicotine stimulates DNA synthesis and proliferation in vascular endothelial cells in vitro. J Appl Physiol 84: 2089-2098, 1998.

20. Conklin BS, Zhao W, Zhong DS and Chen C: Nicotine and cotinine up-regulate vascular endothelial growth factor expression in endothelial cells. Am J Pathol 160: 413-418, 2002.
21. Heeschen C, Jang JJ, Weis M, Pathak A, Kaji S, Hu RS, Tsao PS, Johnson FL and Cooke JP: Nicotine stimulates angiogenesis and promotes tumor growth and atherosclerosis. Nat Med 7: 833-839, 2001.

22. Heeschen C, Weis M, Aicher A, Dimmeler S and Cooke JP: A novel angiogenic pathway mediated by non-neuronal nicotinic acetylcholine receptors. J Clin Invest 110: 527-536, 2002.

23. Guo P, Hu B, Gu W, Xu L, Wang D, Huang HJ, Cavenee WK and Cheng SY: Platelet-derived growth factor-B enhances glioma angiogenesis by stimulating vascular endothelial growth factor expression in tumor endothelia and by promoting pericyte recruitment. Am J Pathol 162: 1083-1093, 2003.

24. Guo P, Xu L, Pan S, Brekken RA, Yang ST, Whitaker GB, Nagane M, Thorpe PE, Rosenbaum JS, Su Huang HJ, Cavenee WK and Cheng SY: Vascular endothelial growth factor isoforms display distinct activities in promoting tumor angiogenesis at different anatomic sites. Cancer Res 61: 8569-8577, 2001.

25. Cheng SY, Nagane M, Huang HS and Cavenee WK: Intracerebral tumor-associated hemorrhage caused by overexpression of the vascular endothelial growth factor isoforms VEGF121 and VEGF165 but not VEGF189. Proc Natl Acad Sci USA 94: 12081-12087, 1997.

26. Concina P, Sordello S, Barbacanne MA, Elhage R, Pieraggi MT, Fournial G, Plouet J, Bayard F and Arnal JF: The mitogenic effect of 17beta-estradiol on in vitro endothelial cell proliferation and on in vivo reendothelialization are both dependent on vascular endothelial growth factor. J Vasc Res 37: 202-208, 2000.

27. Suzuma I, Mandai M, Takagi H, Suzuma K, Otani A, Oh H, Kobayashi $\mathrm{K}$ and Honda Y: 17 Beta-estradiol increases VEGF receptor-2 and promotes DNA synthesis in retinal microvascular endothelial cells. Invest Ophthalmol Vis Sci 40: 2122-2129, 1999.

28. Buteau-Lozano H, Ancelin M, Lardeux B, Milanini J and Perrot-Applanat M: Transcriptional regulation of vascular endothelial growth factor by estradiol and tamoxifen in breast cancer cells: a complex interplay between estrogen receptors alpha and beta. Cancer Res 62: 4977-4984, 2002.

29. Lee JE, Chung KW, Han W, Kim SW, Kim SW, Shin HJ, Bae JY and Noh DY: Effect of estrogen, tamoxifen and epidermal growth factor on the transcriptional regulation of vascular endothelial growth factor in breast cancer cells. Anticancer Res 24: 3961-3964, 2004.

30. Ruohola JK, Valve EM, Karkkainen MJ, Joukov V, Alitalo K and Harkonen PL: Vascular endothelial growth factors are differentially regulated by steroid hormones and antiestrogens in breast cancer cells. Mol Cell Endocrinol 149: 29-40, 1999.

31. Sengupta K, Banerjee S, Saxena N and Banerjee SK: Estradiolinduced vascular endothelial growth factor-A expression in breast tumor cells is biphasic and regulated by estrogen receptor-alpha dependent pathway. Int J Oncol 22: 609-614, 2003.

32. Plummer HK III, Dhar M and Schuller HM: Expression of the alpha7 nicotinic acetylcholine receptor in human lung cells. Respir Res 6: 29, 2005.

33. Castro-Rivera E, Ran S, Thorpe P and Minna JD: Semaphorin 3B (SEMA3B) induces apoptosis in lung and breast cancer, whereas VEGF165 antagonizes this effect. Proc Natl Acad Sci USA 101: 11432-11437, 2004. 